\title{
The WINNER B3G System MAC Concept
}

\author{
Mikael Sternad $^{*}$, Tommy Svensson ${ }^{\dagger}$ and Göran Klang ${ }^{\#}$
}

*Signals and Systems, Uppsala University, Uppsala, Sweden. E-mail: mikael.sternad@signal.uu.se
${ }^{\dagger}$ Dept. Signals and Systems, Chalmers, Göteborg, Sweden. E-mail: tommy.svensson@chalmers.se
\#Ericsson Research, Ericsson AB, Stockholm, Sweden. E-mail: goran.n.klang@ericsson.com

Abstract - The European IST research projects WINNER and WINNER II aim at developing a single new ubiquitous radio access system concept that can be adapted for use in a wide variety of mobile communication scenarios, from wide area coverage to hot spots. This cellular packet data system uses a multicarrier-based air interface. Its medium access control (MAC) system layer is designed for either FDD or TDD cellular transmission. It supports adaptive transmission by tight interaction with the physical layer, and provides efficient resource allocation and scheduling within relay-enhanced cells. It also supports separate allocation of antenna resources per packet flow and furthermore provides support for spectrum sharing and flexible spectrum use. The paper outlines the resource allocation within a super-frame and the use of either opportunistic scheduling or diversity-based transmission within frames.

Index Terms -WWI-Session, Wireless resource management, WINNER, Medium Access Control, mobile wireless systems, radio resource control, OFDM.

\section{INTRODUCTION}

The European WINNER research projects are a cooperation of more than 40 partners from industry, operators, and academia, partly funded by the European Union. They have the overall goal to develop a single radio interface that covers a range of scenarios from isolated hot spots to wide area cellular deployment, by using different modes of a common technology [1], [10]. The targets are increased data rates, low latency, and high system capacity. The main tools used are adaptive transmission, flexible spectrum usage, relaying, and advanced multi-antenna processing. The phase 1 of the WINNER project (2004-2005) evaluated technologies and combined them into a system concept. It forms the basis for the WINNER II project (2006-2007), which aims at a detailed design and assessment of a beyond $3 \mathrm{G}$ system proposal. WINNER II develops and evaluates the system concept in three deployment scenarios: Wide area cellular deployment, metropolitan area cellular systems, with up to $500 \mathrm{~m}$ cell radius and local area deployment. The projects are part of the Wireless World Initiative (WWI), a set of cooperating EU Integrated Projects.

The WINNER radio interface design is aimed at attaining both high flexibility in terms of spectrum use and fulfillment of user requirements and a high spectral efficiency; two goals that are often contradictory and difficult to combine. The medium access control (MAC) system layer plays an important role for fulfilling these goals. It controls the physical layer and the radio resource allocation, the spatial processing and the packet scheduling. In existing systems, these tasks are associated with Logical Link Control, Medium Access Control and Radio Resource Control protocol (sub)layers. The present paper focuses on the main resource allocation problems that are to be solved, outlines the timefrequency-spatial resource units and the control function blocks designed to solve them. Detailed protocol aspects of the MAC system layer will not be discussed here, and are being developed within WINNER II.

The MAC concept of WINNER

- Supports flexible spectrum use, in spectrum shared by operators who all use one physical-layer WINNER mode [6].

- Offers support for spectrum sharing with e.g. fixed microwave links or satellite systems. This would enable coexistence with legacy systems within frequency bands in the range 3.5-5.0 GHz. These bands have been identified as candidate bands for beyond $3 \mathrm{G}$ systems.

- The resource partitioning is designed to work efficiently in conjunction with inter-cell interference-avoidance schemes. It is designed for relay-enhanced cells, so that base stations (BSs) and layer two relay nodes (RNs) may share the spectral resources.

- A super-frame is designed with pilots that support selforganized network synchronization among base stations, relay nodes and user-terminals (UTs). Ensuring a synchronized network enables improved spectral efficiency in two ways: it makes large guard-bands unnecessary and it simplifies interference avoidance between cells and relay nodes.

- Low transmission latency and very low retransmission delays over the radio interface (around $2 \mathrm{~ms}$ ) are supported [4]. This is key to attaining high spectral efficiency via adaptive schemes, reliable communication through efficient re-transmission and high data rates for TCP/IP traffic.

- Adaptive transmission is integrated into the design, at all time scales. Up to moderate vehicular velocities, link adaptation and scheduling can be performed with fine granularity in the time-frequency domain (TDMA/OFDMA) $[2,8]$. This provides multi-user scheduling gains for mobile as well as stationary terminals. For higher velocities, the transmission only adapts to the path loss and shadow fading. On slower time scales, the resource partitioning adapts to the 
traffic demand.

- Multi-antenna transmission and reception can be adjusted in a very flexible way per packet flow, to obtain an appropriate balance between various objectives: Obtaining multiplexing gains to boost throughput, achieving robustness via diversity transmission, and obtaining SDMA (spatialdivision multiple access) gains by transmitting flows to different user terminals over different spatial channels [7].

Section II below summarizes the WINNER phase I system concept and Section III outlines some aspects of the physical layer. Section IV then outlines the MAC system layer as described by WINNER deliverables D2.10 [2] and D7.6 [6].

\section{SYSTEM LAYERS, MODES AND PARAMETERIZATIONS}

There are four system layers in the WINNER I concept as described in [6], the highest three subdivided into user plane and control plane. Services that need to operate directly on individual packets are located in the user plane.

\section{A. System layers}

\section{IP convergence (IPC)}

The IPC layer receives packets, performs RAN association as well as macro-mobility (IP level mobility). These functions, and the cooperation with legacy RANs, are at present coordinated with the WWI project Ambient Networks.

\section{Radio link control (RLC)}

The user plane of the RLC layer provides reliable packet transfer over the radio interface. It also performs confidentiality protection and packet prioritization in order to meet the quality-of-service (QoS) goals. The control plane takes care of flow establishment and release, location services, load, spectrum, and micro-mobility control.

\section{Medium access control (MAC)}

The MAC system layer will be described in Section IV below.

\section{Physical (PHY) layer}

The PHY system layer handles the physical transmission of flows and of measurements and control signaling directly related to the radio interface. There is a tight inter-layer interaction between MAC and physical layers and this is crucial for the performance of the WINNER system. Some functions, such as encoding and decoding, that are traditionally placed in the physical layer are in the WINNER system concept placed in the MAC layer.

\section{B. Modes and flexible parameterizations}

A basic goal is that the WINNER radio interface should present a unified set of services to higher layers, yet include some specific parts that provide the required flexibility. To provide flexibility and convergence in a structured way, the definition of modes is helpful. A system mode represents a specific combination of physical layer modes (PLM) and $M A C$ modes. All IPC and RLC functions are designed to be mode- independent (generic) and they form the unified interface of the WINNER system.

A separate physical layer mode has been defined where there is a significant impact (discontinuity in adaptation) of PHY functionality. Only two PLMs have so far been required:

- Frequency division duplex (FDD) transmission, performed over paired bands and supporting halfduplex FDD terminals.

- Time division duplex (TDD) transmission over an unpaired band.

- There are three MAC modes within the WINNER concept:

- FDD cellular MAC

- TDD cellular MAC

- MAC for peer-to-peer transmission, at present designed using the TDD physical layer mode.

The two MACs for cellular transmission are largely similar and are described in this paper. The peer-to-peer MAC has been described in [9] and [3].

The combinations of PHY and MAC modes thus define three system modes: FDD cellular, TDD cellular and Peer-to-peer.

Parameterizations within modes provide further flexibility and adaptability. Both PLMs use generalized multi-carrier (GMC) transmission, which includes cyclic prefix-OFDM and serial modulation as special cases [3]. See Section III below. Multiple access is realized in frequency, time, space, and in particular cases also in the code domain [3].

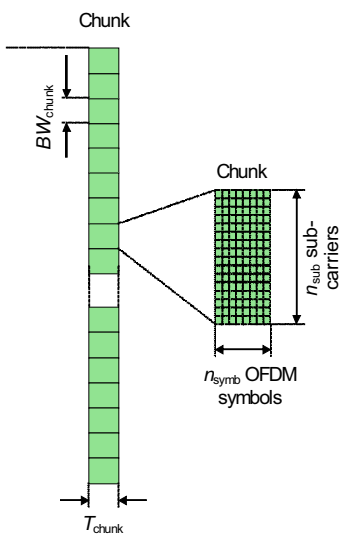

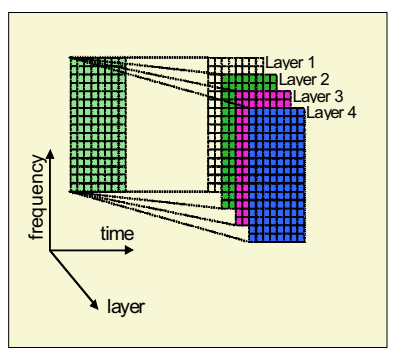

b)
Fig. 1. : a) Physical channel structure and chunks in multicarrier channel. b) Chunk layers obtained by spatial reuse.

The basic time-frequency unit for resource partitioning is denoted a chunk. It consists of a rectangular time-frequency area, see Fig.1a), that contains payload symbols and pilot symbols. The channel variations should be rather small within chunks so that the same link adaptation parameters can be used. The number of offered payload bits per chunk depends on the utilized modulation-coding formats, and on the chunk sizes. Use of multiple antennas is an integral part of the WINNER RI design. Spatial multiplexing may reuse the timefrequency resource defined by the chunk. A chunk layer represents the spatial dimension (Fig. 1b). 


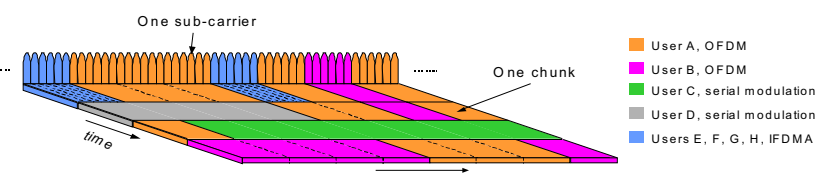

Fig. 2. : Use of different GMC variants by different uplink users.

The chunks and chunk layers are pre-assigned to different classes of data flows, on a super-frame time scale (Section IV below). They are then used in a flexible way to optimize the transmission performance. For example, the available antenna resources can be used differently for different flows to/from user terminals.

\section{The Physical LAYer}

The WINNER physical layer is based on multicarrier transmission. This simplifies a flexible use of spectral resources. After considering different alternatives, standard cyclic prefix OFDM (CP-OFDM) has been selected whenever terminal power consumption is not a critical constraint. For power-constrained uplinks, the baseline scheme is to use a serial modulation waveform, with low peak-to-average power ratio, in the allocated chunks, as illustrated by Fig. 2. This scheme (DFT-precoded CP-OFDM) is obtained by applying an extra DFT at the transmitter and at the receiver [3]. Note that all transmitters are assumed to be synchronized, so other uplink users could use other waveforms in other sets of chunks, such as ordinary OFDM (Fig. 2).

During the later part of WINNER phase I, two particular example designs, a $2 \times 20 \mathrm{MHz}$ FDD scheme and TDD transmission over a $100 \mathrm{MHz}$ unpaired band, were used for assessment by simulations [3]. Table 1 below illustrates the corresponding radio parameters. The WINNER system will also be required to support several other configurations (parameter sets).

Table 1: Basic transmission parameters for simulation

\begin{tabular}{|l|c|c|}
\hline Parameter & FDD mode & TDD mode \\
\hline Centre frequency $(\mathrm{GHz})$ & $5.0 \mathrm{DL} / 4.2 \mathrm{UL}$ & 5.0 \\
\hline Duplexing method & FDD (paired) & TDD \\
\hline FFT bandwidth $(\mathrm{MHz})$ & 20.0 & 100.0 \\
\hline Number of subcarriers in GMC & 512 & 2048 \\
\hline Subcarrier spacing $(\mathrm{Hz})$ & 39062 & 48828 \\
\hline Symbol duration (excl. cycl prefix) $(\mu \mathrm{s})$ & 25.60 & 20.48 \\
\hline Cyclic prefix $(\mu \mathrm{s})$ & 3.20 & 1.28 \\
\hline Number of subcarriers in use & 416 & 1664 \\
\hline Signal bandwidth $(\mathrm{MHz})$ & 16.25 & 81.25 \\
\hline Chunk size (subcarriers x symbols) & $8 \times 12=96$ & $16 \times 5=80$ \\
\hline
\end{tabular}

\section{THE MAC SySTEM LAYER}

\section{A. The MAC services and tasks}

The WINNER system is fully packet oriented. Active packet streams are denoted flows, classified by QoS requirement, source and destination(s). When flows are set up, a set of resources and queues are prepared at the UT, at the BS and possibly also at more centralized nodes.

The MAC system layer for FDD or TDD cellular transmission is assumed to be part of each BS and RN. It controls the radio resource allocation within relay-enhanced cells (see IV-B below) and transmission over one hop within such cells: Between BS and RN, between RN and UT and directly between a BS and a UT. Flow QoS control over links spanning multiple hops is the responsibility of the RLC layer.

The MAC system layer has a set of MAC protocols implemented in its user plane. It also contains resource allocation and planning functions in its control plane.

The user-plane interface to higher layers will be provided by a set of logical channels, which classify the type of information (e.g. one-to-one user data, one-to-many user data, control). The logical channels are mapped onto transport channels that define the basic types of radio-packet transfer, such as random access or scheduled traffic. Transport channels are then mapped on different physical locations within the super-frame, as described in Section IV-C below. The WINNER design contains the following transport channels:

- Broadcast channel (BCH) for broadcasting system information from RLC and higher layers to all terminals inside the coverage area of the cell,

- Contention-based random access channel (RAC), used for initial access to a base station (BS) or relay node (RN), and also for BS-to-BS control signaling in the TDD mode,

- Contention-based direct access channel (DAC) for contention-based uplink data transfer,

- Common data channel (CDC) for scheduled point-tomultipoint communication,

- Targeted data channel (TDC) for scheduled point-topoint communication,

- Targeted control channel (TCC) for control-plane generated control messages.

\section{B. Control of relay-enhanced cells}

The MAC system layer is designed to work in relay-enhanced cells (REC), where a set of nodes (RNs or BS) are linked to each other over the air. The BS, but not the RNs, has a fixed connection to the RAN. The RNs may be used for improving the coverage within the cell or for extending the range of the cell [4]. Each RN is connected to one but not more BSs. The RNs essentially control separate sub-cells.

The cellular MAC design has so far mainly focused on the case of homogenous relaying, where RNs and BS use the same PLM and thus have to share the spectral resources. The total time-frequency resources are then partitioned into parts used by the BS, shared parts, and parts used by RNs $[3,4]$.

\section{The chunk, slot, frame and super-frame structure}

As outlined in Section II-B, the basic time-frequency unit for resource allocation is the chunk. In the FDD physical layer mode described in Table 1, chunks comprise 8 subcarriers by 12 OFDM symbols or $312.5 \mathrm{kHz} \times 345.6 \mu \mathrm{s}$. In the TDD physical layer mode, mainly evaluated in short-range scenarios, the chunks are wider in frequency, with dimension 16 subcarriers by 5 symbols, or $781.25 \mathrm{kHz} \times 108.0 \mu \mathrm{s}$. 

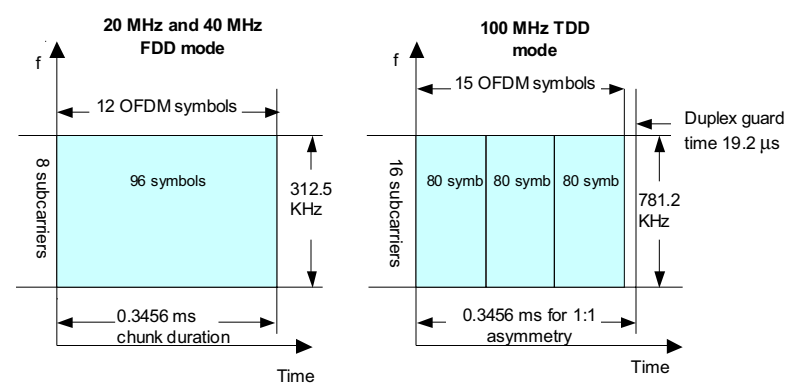

Fig. 3. : Summary of assumed chunk sizes in the two physical layer modes. A slot (half of the frame) is shown in each case, assuming 1:1 TDD asymmetry.

The chunks are organized into frames. In the TDD mode, each frame consists of a downlink transmission interval followed by an uplink transmission interval, denoted slots, or time-slots. In FDD, the frame is also split into two slots. Half-duplex terminals may be separated into two groups, where one group has downlink transmission in the first slot and transmits in uplinks in the other, while the other group uses the opposite scheme. FDD base stations use full duplex and full duplex terminals are also supported.

The frame duration has been set equal in the two PLMs, to facilitate inter-mode cooperation. With a frame duration of $691.2 \mu \mathrm{s}$, an FDD frame consists of two chunk durations, with one chunk per slot. A TDD frame consists of in total 6 chunks and two duplex guard intervals, organized into a downlink slot and an uplink slot. With downlink-uplink asymmetry $1: 1$, the TDD slot thus consists of three downlink chunks followed by three uplink chunks. See Fig. 3.

The super-frame (SF) is a time-frequency unit that contains pre-specified resources for all the transport channels. Fig. 4 illustrates its preliminary design, comprising of a preamble followed by $n_{f}$ frames. In the example, $n_{f}=8$, resulting in super-frames of duration $5.6 \mathrm{~ms}$.

The super-frame is synchronous in all BS and RN. In the FDD mode, a separate super-frame is required in each of the paired bands. It is assumed that there exist some frequency bands allocated to WINNER that are available everywhere. The super-frame preamble is transmitted such a commonly available frequency band. It has the following structure:

- It contains two synchronization slots. Self-organizing synchronization of terminals and network nodes, as described in [3] can be used on a super-frame basis by this design.

- In-between the synchronization slots, a short timeslot over the whole band is reserved for the contention-based random access channel (RAC), plus a guard time.

- Subsequently, a set of OFDM symbols contains the broadcast control channel $(\mathrm{BCH})$ messages from the RLC layer. It also contains a control message that specifies the over-all resource allocation used within this super-frame.

The remaining $n_{f}$ frames may use the commonly available frequency band and may also use other bands that are available at some locations, or to some operators, but not to others. All bands are spanned by one FFT at the receiver and are at present assumed to span at most $100 \mathrm{MHz}$. This part of the super-frame is shared by the contention-based direct access channel (DAC), the scheduled data channels CDC and TDC, and their related control signaling. The DAC resource is used both for the DAC channel and for peer-to-peer transmission [9] in short-range deployments. It is organized as a constant set of frequencies over the whole super-frame, to enable the use of carrier-sense multiple access-based transmission.

Time-frequency resource partitioning and spatial scheme control is planned on a time scale of the super-frame. The allocation to different transport channels is adjusted on this time scale, based on the aggregated demand. Specification of unused chunks enables flexible spectrum use between WINNER operators/users [5] and adaptive interference avoidance between cells/parts of cells.

The definition of unused chunks is performed by a spectrum mapping and assignment function at the RLC layer (Fig. 5), in cooperation with a constraint processing function at the MAC layer. The outcomes of this processing are tables defining restrictions on the use of chunks in specific spatial directions by the resource scheduler at the MAC layer, described below.

The definition and use of time-frequency-spatial guard regions is important for single-system spectrum sharing. It also enables spectrum coexistence with legacy systems, for example microwave links.

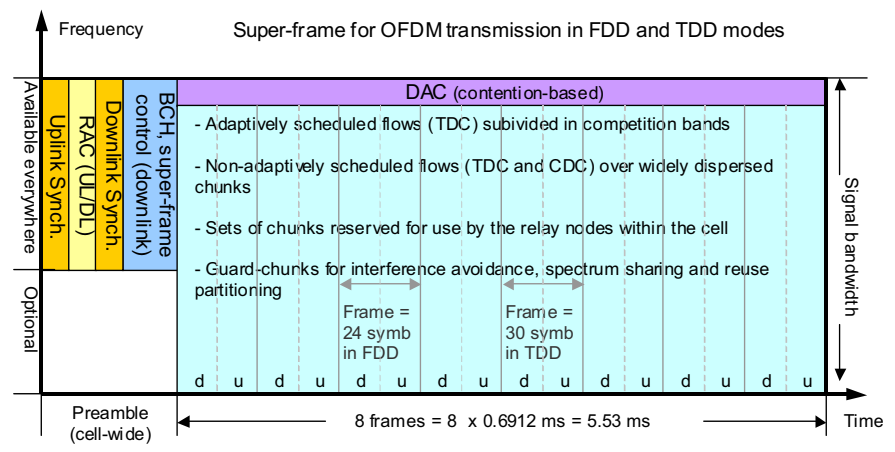

Fig. 4. : Preliminary super-frame structure for both FDD and TDD physical layer modes. $\mathrm{u}=$ uplink transmission in TDD and $\mathrm{d}=$ downlink in TDD.

\section{Packet segmentation and scheduling}

Fig. 5 below gives an overview of the downlink transmission of scheduled flows (CDC, TDC). Two scheduling entities control the flows: A Flow scheduler at the RLC system layer is part of the Service level controller (SLC) that balances the QoS requirements of different flows.

The Resource scheduler (RS) at the MAC system layer determines the resource mapping for TDC and CDC flows on a slot time-scale. It utilizes two scheduling algorithms:

- The Adaptive resource scheduler, used for highperformance TDC transmission.

- The Non-frequency adaptive resource scheduler, used for all CDC flows and as a fallback alternative for TDC flows. It is also foreseen to be used for parts of the control signaling. 


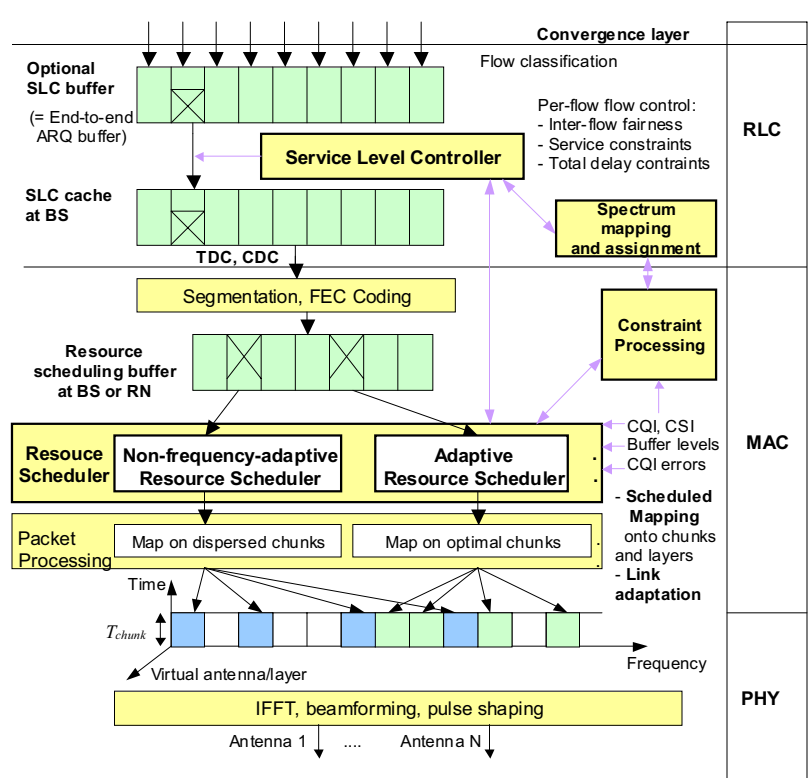

Fig. 5. : Data flows and some control functions in downlinks of the scheduled data channels

The SLC may be implemented at a centralized location. In downlinks, a buffer, the SLC Cache with per-flow buffering, is assumed present in the MAC (at a BS or $\mathrm{RN}$ ). In the present design, these two schedulers, SLC and RS, interact in a very simple way: The SLC fills the SLC Cache, while the RS is responsible for draining it. The SLC furthermore reports priorities of each flow to the RS. Only a few priority levels are used. The RS drains the queues and allocates the transmission resources in a way that optimizes a criterion that takes the queue levels and the priorities into account. Existing algorithms with very low computational complexity, such as weighted fair queuing, maximum weight matching or proportional fair scheduling, can be used to solve the resource scheduling problem, when formulated in this way. These algorithms have computational complexity that grows linearly with the number of scheduled resources and linearly with the number of scheduled flows.

When the Radio Link Control (RLC) Protocol Data Units (PDUs) are entering the MAC, they could be segmented, depending on their size, into ReTransmission Units (RTUs) appropriate for the ARQ. After attachment of CRC, these RTUs are then optionally FEC encoded with an outer code, bit-interleaved and punctured to produce incremental redundancy for the later use by a Hybrid ARQ scheme.

The optionally encoded segments are queued per flow in the resource scheduling buffer. These queues are then drained with bit-level granularity. For each buffer, there is one resource scheduler that determines which queues are to be drained, and to what extent. The resource scheduler works on the time-scale of the chunk duration. The resource scheduler optimizes the allocation of physical channel resources under certain constraints such as interference avoidance. Since adaptive transmission is feasible up to a limiting velocity (around $70 \mathrm{~km} / \mathrm{h}$ ) or down to a certain average user SINR [8], the resource scheduler performs adaptive scheduling when feasible. Otherwise, non-frequency-adaptive scheduling uses averaging w.r.t. frequency-selective channel variations by mapping onto sub-carriers in well-dispersed chunks.

The scheduled bits are mapped either directly by adaptive modulation or adaptive coding and modulation with bit interleaving onto the physical channel resource units, i.e. chunks (in general chunk layers when more than one spatial dimension is utilized in multi-antenna transmitters [7]).

The design outlined above performs segmentation and encoding before the mapping onto chunks. It thus decouples the segment size used for retransmission from the chunk size. It also decouples the code block size from the chunk size. This enables the evaluation of various schemes that combine outer coding, link adaptation that may involve inner coding, and hybrid-ARQ mechanisms.

\section{CONCLUSIONS}

The MAC design outlined above aims to support a radio interface to be both efficient and flexible in terms of user requirements, spectrum use, deployment aspects and terminal capabilities. Within the WINNER II project the basic concept is now developed further by detailed design and comparison of specific protocol features.

\section{ACKNOWLEDGMENT}

This work has been performed in the framework of the IST project IST-2003-507581 WINNER, which is partly funded by the European Union. The authors would like to acknowledge the contributions of their colleagues. In particular, beside the authors, the following researchers have contributed to the WINNER MAC for FDD and TDD Cellular Transmission: Niklas Johansson, Ericsson, Uwe Schwark, BenQ, Jörn von Häfen, Siemens, Martin Döttling, Siemens, Antti Sorri, Nokia, Keith Roberts, Philps, Martin Fuchs, TU Ilmenau, Mats Bengtsson, KTH and Daniel Schulz, Ole Klein and Ralf Pabst, RWTH Aachen University.

\section{REFERENCES}

[1] WINNER, Wireless World Initiative New Radio, www.ist-winner.org

[2] IST-2003-507581 WINNER, "D2.4 Assessment of adaptive transmission technologies". February 2005.

[3] IST-2003-507581 WINNER, "D2.10 Final report on identified RI key technologies, system concept, and their assessment". December 2005.

[4] IST-2003-507581 WINNER, "D3.5 Proposal of the best suited deployment concepts chosen for the identified scenarios", Dec. 2005.

[5] IST-2003-507581 WINNER, "D6.3 WINNER Spectrum Aspects: Assesment report". December 2005.

[6] IST-2003-507581 WINNER, "D7.6 WINNER System Concept Description". October 2005.

[7] M. Döttling, M. Sternad, G. Klang, J. Von Häfen and M. Olsson, "Integration of spatial processing in the WINNER B3G Air interface design," IEEE VTC 2006-Spring, Melbourne, May 2006.

[8] M. Sternad, S. Falahati, T. Svensson and D. Aronsson, "Adaptive TDMA/OFDMA for wide-area coverage and vehicular velocities," IST Mobile Summit. Dresden, July 2005.

[9] P. Coronel and W. Schott, "WINNER MAC for peer-to-peer transmission," IST Mobile Summit, Myconos, June 2006.

[10] A. Pollard, M. Döttling, J. von Häfen, D. Schultz, R. Pabst, E. Zimmerman, "WINNER - Towards ubitiquous wireless access," IEEE VTC 2006-Spring, Melbourne, May 2006. 